Abbreviated Key Title: Sch J Agric Vet Sci

ISSN 2348-8883 (Print) | ISSN 2348-1854 (Online)

Journal homepage: https://saspublishers.com/sjavs/

\title{
Preparation of Acetyl Salicylic Acid by Ultrasonic Collaborative Method
} Jinling Gao*, Jinqiu Ji

\author{
Collage of Science, Heilongjiang Bayi Agricultural University, Daqing, 163319, China
}

DOI: $10.36347 /$ sjavs.2020.v07i03.002

| Received: 10.03.2020 | Accepted: 17.03.2020 | Published: 21.03.2020

*Corresponding author: Jinling Gao

Abstract

Original Research Article

Acetyl salicylic acid was synthesized by the method of microwave radiation, Acetyl chlorine and salicylic acid were used as raw materials, iodine was used as catalyst. The amounts of acetyl chloride, iodine and catalytic time of microwave were investigated on the yield of acetylsalicylic acid, the best test conditions were obtained by orthogonal experiments furthermore. In order to investigate the quality of the products, the products were then applied at the bud of soybean seed. The factors that affect the yield of acetyl salicylic acid are: acetyl chloride (A) >catalytic time of Microwave (C) >catalyst of iodine(B), the amount of salicylic acid is $2.7 \mathrm{~g}$, the amount of acetyl chloride is $2.4 \mathrm{~mL}$, and the amount of iodine is $0.4 \mathrm{~g}$, The catalytic time of microwave is $50 \mathrm{~s}$ at $800 \mathrm{~W}$. Under the above test conditions, the power of acetylsalicylic acid was obtained with the yield of $64.52 \%$, it is odorless and slightly sour.

Keywords: Acetyl salicylic acid; Iodine; Microwave radiation.

Copyright @ 2020: This is an open-access article distributed under the terms of the Creative Commons Attribution license which permits unrestricted use, distribution, and reproduction in any medium for non-commercial use (NonCommercial, or CC-BY-NC) provided the original author and source are credited.

\section{INTRODUCTION}

Acetyl salicylic acid, which also known as aspirin, is prepared by salicylic acid and acetyl chloride [1]. It can play the role of analgesia, anti-inflammatory and cooling in medical [2], at the same time, acetyl salicylic acid has been widely used in agriculture too, as a kind of biological signal molecules, it can identify signal in the course of transduction, it can improve the activity of young seedlings, the quality of seedling can be significantly increased as the using of acetylsalicylic acid in the period of germination. It can help the crops resist drought for a long time, induce plant flowering, extend plant flowering, and increase the yield of fruit. Therefore, acetyl salicylic acid plays an important role in agricultural production [3], it has a critical value on the preparation of acetyl salicylic acid.

Salicylic acid and acetic anhydride (acetylchloride) are catalyzed by concentrated sulfuric acid, acetylsalicylic acid is obtained during the traditional preparation, the yield is about $50 \%$. The catalysis of sulfuric acid is a classical mature method, but the equipment are seriously corroded, the process is complex, the catalyst is difficult to recycle, and environment is polluted because of a large number of waste water [4]. In recent years, $\mathrm{AlCl}_{3}$, solid superacid, acidic ionic liquid, citric acid, vitamin $\mathrm{C}$, molecular iodine, strongly acidic cation exchange resin and molecular sieve were used as catalysts in the synthesis of acetylsalicylic acid, comparing with the traditional sulfuric acid, this kind of catalytic reaction is relatively mild [5]. The research shew that $\mathrm{Y}_{2} \mathrm{P}_{2} \mathrm{~W}_{18} \mathrm{O}_{62} \cdot \mathrm{nH}_{2} \mathrm{O}$ can be used as catalyst to prepare acetyl salicylic acid, the catalyst can be recycled for 6 times, and the yield is about $75 \%$, but the preparation and purification of catalyst is complicated [6]. Ultrasonic technology was also used in the synthesis of acetyl salicylic acid, it can shorten reaction time [7]. $\mathrm{PWMo}_{11}$-LDHs can be used as catalyst to prepare acetyl salicylic acid, and the yield is about $80 \%$, but the circulation performance of the catalyst was not good [8]. Ascorbic acid can be used as catalyst to prepare acetyl salicylic acid, and the yield is about $85 \%$ [9]. Based on the above analysis, iodine was used as catalyst, acetyl salicylic acid was synthesized under the conditions of microwave coordination in this paper [10].

\section{MATERIALS AND INSTRUMENTS Experimental Reagents}

The salicylic acid, acetyl chloride, iodine, sodium bicarbonate and $95 \%$ ethanol are analytically pure, the concentrated hydrochloric acid are chemically pure.

\section{Instruments and Equipment}

Electronic analytical balance (ALC-310.2), jinghai instrument co. LTD of Shanghai. The microwave oven (MM721AAU-PW), midea microwave manufacturing co. LTD of Guangdong. Circulating water and multi-purpose vacuum pump (SHB-IIIA), Great Wall science and trade co. LTD of Zhengzhou. 
Jinling Gao \& Jinqiu Ji., Sch J Agric Vet Sci, March, 2020; 7(3): 61-64

Ultraviolet spectrophotometer (T6), puxi tongyong instrument co. LTD of Beijing. Fourier transform infrared spectrometer (Nicolet IS5), Thermofisher. Micromelting point tester (X-8), taike instrument co. LTD of Beijing.

\section{METHODS}

\section{The Preparation of saturated sodium bicarbonate solution}

Sodium bicarbonate $(9.8000 \mathrm{~g})$ was weighed and added to a beaker $(250 \mathrm{~mL})$, distilled water $(100.0$ $\mathrm{ml}$ ) was added to the beaker, when sodium bicarbonate was no longer dissolved in water, it indicated that the solution was saturated, the solution of saturated sodium bicarbonate was prepared.

\section{The Preparation of Acetyl salicylic acid}

Salicylic acid $(2.7000 \mathrm{~g})$ and acetyl chloride $(1.60 \mathrm{~mL})$ were put a dried conical flask, iodine $(0.4000$ g) was put as catalyst then. The solution was shaken well and put in a microwave oven, the reaction was reacted for $40 \mathrm{~s}$ at $800 \mathrm{~W}$, the reaction was stopped after being cooled, and distilled water $(2.00 \mathrm{~mL})$ was added to the conical flask, the excess acetyl chloride was broken down, then the solution was cooled to room temperature. After the crystallization was fully precipitated, distilled water $(50.0 \mathrm{ml})$ was added and cooled with ice water, then a large amount of solid was precipitated. At last, the solution was filtrated, the coarse product of acetyl salicylic acid was obtained.

\section{The Purification of acetyl salicylic acid}

The product, which obtained in the previous step, was placed in a beaker $(250 \mathrm{ml})$, the saturated solution of sodium bicarbonate $(70.0 \mathrm{ml})$ was added to the beaker, the solution was continuously stirred with a glass rod until the gas of $\mathrm{CO}_{2}$ was not released. The insoluble polymer solids were removed by a method of filtration with the filter device, the filtrate was put into a beaker $(250 \mathrm{ml})$, and $\mathrm{HCl}(6 \mathrm{~mol} / \mathrm{L})$ solution was slowly added. The solution was cooled until the crystallization was completely precipitated. The purified product of acetyl salicylic acid was obtained after the solution was filtrated. Since the group of phenolic hydroxyl is not present in acetylsalicylic acid, it is possible to determine whether the hydroxyl group of salicylic acid has been acylated in the reaction. The synthesized product was tested with the solution of ferric chloride, and its purity could be roughly determined by observing whether the color turned purple.

\section{The test of single factor}

Salicylic acid and acetyl chloride were used as materials, iodine was used as catalyst, the acetyl salicylic acid was synthesized. The amount of acetyl chloride $(1.60,2.40,3.20 \mathrm{~mL})$, The amount of catalyst $(0.2000,0.4000,0.6000 \mathrm{~g})$, and the time of reaction $(30$, $40,50 \mathrm{~s})$ were inspected to determine the optimum condition respectively.

\section{The design of orthogonal experiment}

In order to determine the optimal experimental conditions, on the basis of single factor experiment, three-factor and three-level orthogonal experiment were further designed to optimize the conditions of experiments. The specific data were shown in Table-1.

Table-1: The table of orthogonal factors and levels

\begin{tabular}{|l|l|c|c|}
\hline level & $\begin{array}{l}\text { The amount of acetyl } \\
\text { chloride(A)/mL }\end{array}$ & $\begin{array}{l}\text { The amount of } \\
\text { catalyst content(B)/g }\end{array}$ & $\begin{array}{l}\text { The time of } \\
\text { catalytic time(C)/s }\end{array}$ \\
\hline 1 & 1.60 & 0.2000 & 30 \\
\hline 2 & 2.40 & 0.4000 & 40 \\
\hline 3 & 3.20 & 0.6000 & 50 \\
\hline
\end{tabular}

\section{RESULTS AND ANALYSIS}

The effect of acetyl chloride on the yield of acetyl salicylic acid

The relationship between the amount of acetyl chloride and the yield of acetyl salicylic acid was listed in Table2. As is shown in Table-2, it can be determined that the optimal amount of acetyl chloride is $2.40 \mathrm{~mL}$.

Table-2: The influence of acetyl chloride on product yield

\begin{tabular}{|l|l|l|l|}
\hline Acetyl chloride (mL) & 1.60 & .40 & 3.20 \\
\hline The yield of acetyl salicylic acid (\%) & 6.31 & 4.74 & 4.29 \\
\hline
\end{tabular}

The effect of iodine on the yield of acetyl salicylic acid

The relationship between the amount of iodine and the yield of acetyl salicylic acid was listed in Table-
3. As is shown in Table-3, it can be determined from the final results that the optimum addition of iodine is $0.4 \mathrm{~g}$.

Table-3: The influence of iodine on the yield of product

\begin{tabular}{|l|l|l|l|}
\hline The content of catalyst $(\mathrm{g})$ & .2000 & .4000 & .6000 \\
\hline The yield of acetyl salicylic acid (\%) & 5.56 & 8.68 & 0.63 \\
\hline
\end{tabular}


The time of catalytic on the yield of acetyl salicylic acid

The relationship between the time of reaction and the yield of acetyl salicylic acid was listed in Table-
4. As is shown in Table-4, the optimum time of catalysis is $50 \mathrm{~s}$.

Table-4: The influence of catalytic time on the yield of product

\begin{tabular}{|l|l|l|l|}
\hline The time of catalytic (s) & 0 & 0 & 0 \\
\hline The yield of acetyl salicylic acid (\%) & 4.37 & 2.64 & 8.12 \\
\hline
\end{tabular}

\section{The results and analysis of orthogonal test}

On the basis of single-factor experiments, the orthogonal experiments of $\mathrm{L}_{9}\left(3^{3}\right)$ were designed, the experiment was proceeded according to $\mathrm{L}_{9}\left(3^{3}\right)$, the maximum yield of acetylsalicylic acid was obtained. The data obtained was shown in Table-5. According to the orthogonal Table-5, the acetyl salicylic acid product was obtained.

Table-5: Results and analysis of $L_{9}\left(3^{3}\right)$ orthogonal experiments

\begin{tabular}{|l|l|l|l|l|l|}
\hline Number & A & Null & B & C & $\mathbf{y}$ \\
\hline 1 & 1 & 1 & 1 & 1 & 26 \\
\hline 2 & 1 & 2 & 2 & 2 & 28 \\
\hline 3 & 1 & 3 & 3 & 3 & 41 \\
\hline 4 & 2 & 3 & 1 & 2 & 50 \\
\hline 5 & 2 & 1 & 2 & 3 & 64 \\
\hline 6 & 2 & 2 & 3 & 1 & 36 \\
\hline 7 & 3 & 2 & 1 & 3 & 18 \\
\hline 8 & 3 & 3 & 2 & 1 & 15 \\
\hline 9 & 3 & 1 & 3 & 2 & 11 \\
\hline K1 & 95 & 101 & 94 & 77 & \\
\hline K2 & 150 & 82 & 107 & 89 & \\
\hline K3 & 44 & 106 & 88 & 123 & \\
\hline k1 & 31.67 & 33.66 & 31.33 & 25.67 & \\
\hline k2 & 50.00 & 27.33 & 35.67 & 29.67 & \\
\hline k3 & 14.67 & 35.33 & 29.33 & 41.00 & \\
\hline R & 35.33 & 6.33 & 6.33 & 15.33 & \\
\hline
\end{tabular}

Three parallel experiments were carried out according to the best method of producing acetylsalicylic acid obtained in the previous step. The acetylsalicylic acid (2.3228g) was obtained, the average yield of acetylsalicylic acid is $64.52 \%$, and the relative deviation of the results is small, a method for the preparation of acetylsalicylic acid was obtained. The melting point of the obtained acetylsalicylic acid powder was determined, its melting process was determined at $137-140{ }^{\circ} \mathrm{C}$, and the standard deviation is small, it is illustrated that the purity of the product is higher.

\section{The analysis of Infrared spectroscopy}

In order to further investigate the structure of the product, the infrared spectroscopy was conducted. As is shown in Figure-1, the curve a is the Infrared spectrum of standard acetylsalicylic acid, and the curve b is the Infrared spectrum of obtained produce.

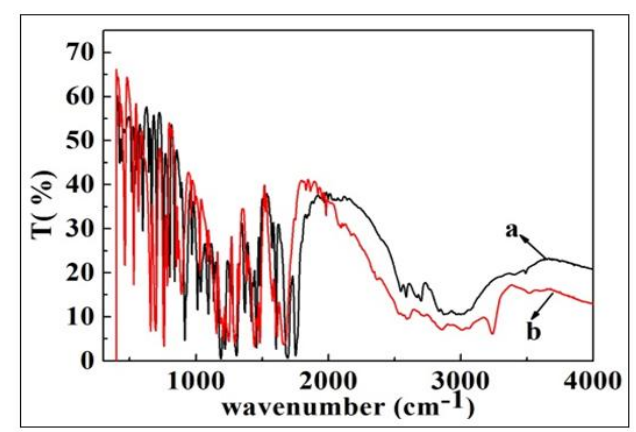

Fig-1: Infrared spectrum of standard acetylsalicylic acid (a) and obtained produce (b)

It can be seen from the curve $b$, the peak of methyl bond is vibrated at $3000 \mathrm{~cm}^{-1}$, the peak of $\mathrm{CH}$ bond of benzene ring is vibrated at $2658 \mathrm{~cm}^{-1}$, the peak of bond of $\mathrm{OH}$ group is vibrated at $2547 \mathrm{~cm}^{-1}$, the peak of $\mathrm{C}=\mathrm{O}$ bond of the ester bond is vibrated at $1749 \mathrm{~cm}^{-1}$, the peak of $\mathrm{C}=\mathrm{O}$ bond of carboxyl group is vibrated at $1678 \mathrm{~cm}^{-1}$, the peak of $\mathrm{C}=\mathrm{C}$ bond in the benzene ring skeleton is vibrated at $1482 \mathrm{~cm}^{-1}$. In general, the peaks of curve $b$ is consistent with those of curve $a$, it can be confirmed that acetylsalicylic acid was synthesized successfully.

\section{The analysis of UV spectroscopy}

In order to investigate the purity of the product, Uv analysis was conducted further. As is shown in Figure-2, the maximum absorption wavelength of standard acetylsalicylic acid solution was at $300 \mathrm{~nm}$.

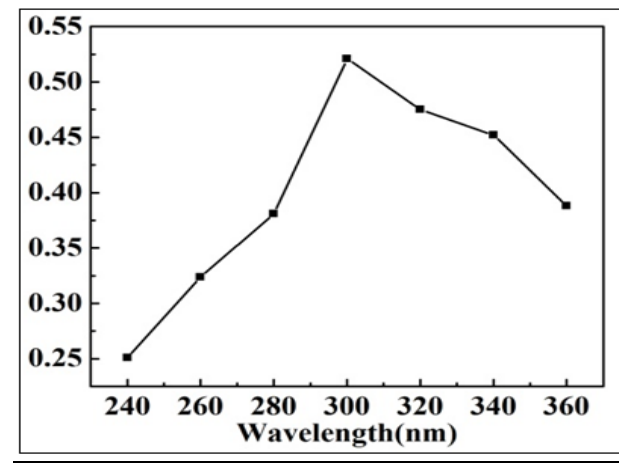

Fig-2: The maximum absorption wavelength of the sodium salicylate solution

The standard solutions of different concentrations were prepared, and the absorbance of different standard solutions were measured at $300 \mathrm{~nm}$, and the absorbance was obtained. According to the absorbance, the standard curve was drawn as shown in Figure-3. 


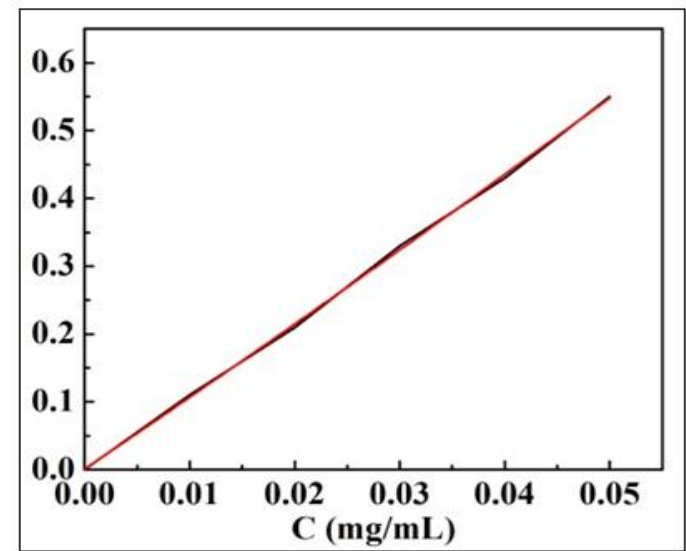

Fig-3: The standard curve of the sodium salicylate solution

In order to inspect the reliablity of the absorbance, linear fitting was operated, and the equation of standard curve was obtained as formula (1). $\mathrm{A}=11 \mathrm{C}-0.0003, \mathrm{R}^{2}=0.99921$
It is shown that the content of sodium salicylate and the absorbance have a good linear relationship. Combining with the equation of the standard curve, the content of sodium salicylate can be calculated as formula (2).

$$
\mathrm{W}=(\mathrm{C} \cdot \mathrm{V} \cdot \mathrm{M}) / \mathrm{m}
$$

In the formula, $\mathrm{W}$ is Mass percentage of sodium salicylate (\%), C is the concentration calculated by the standard curve $(\mathrm{mg} / \mathrm{mL}), \mathrm{V}$ is the volume of the extraction $(\mathrm{mL}), \mathrm{m}$ is the mass of sample $(\mathrm{g})$.

\section{Effect of acetyl salicylic acid on seed germination}

In order to investigate the effect of acetyl salicylic acid on seed germination, the seeds of soybean were researched, a comparative experiment was conducted, a amount of acetyl salicylic acid was added in the solution of the seeds of soybean, and the other group without adding acetyl salicylic acid. The results were shown in Table-6.

Table-6: Variation of average sprouts length of soybeans

\begin{tabular}{|l|l|l|l|l|}
\hline Number & 1 & 2 & 3 & 4 \\
\hline average sprouts length of soybeans with sodium salicylate $\mathbf{( c m )}$ & 6.00 & 6.50 & 8.00 & 6.40 \\
\hline average sprouts length of soybeans without sodium salicylate $\mathbf{( c m )}$ & 5.78 & 6.31 & 7.66 & 6.09 \\
\hline
\end{tabular}

The results showed that acetyl salicylic acid could promote the seed germination of crops, and it could be applied to agriculture to increase the yield of crops, which was of great significance to agricultural production.

\section{CONCLUSION}

Acetyl salicylic acid was synthesized by the method of microwave radiation. On the basis of the test of single factor, the experiments of three-factors and three-levels were designed, the test of orthogonal was carried out to determine the optimal synthesis conditions for acetyl salicylic acid, acetyl chlorine and salicylic acid were used as raw materials, iodine was used as catalyst. The amounts of salicylic acid, acetyl chloride and iodineis are $2.7 \mathrm{~g}, 2.4 \mathrm{~mL}$ and $0.4 \mathrm{~g}$, the catalytic time of microwave is $50 \mathrm{~s}$ at $800 \mathrm{~W}$. The power of acetylsalicylic acid was obtained with the yield of $64.52 \%$, it is odorless and slightly sour. The process is simple, reproducible, economical and feasible. The acetylsalicylic acid, which obtained in this experiment, can promote the seed germination of crops, which can be gradually applied to agriculture, and it is of great significance to production of agriculture.

\section{REFERENCES}

1. Montinari MR, Minelli S. The first 3500 years of aspirin history from its roots-A concise summary [J]. Higher science education, 2016, 107-113.

2. Stellar W. Synthesis and Application of Acetylsalicylic Acid [J]. Private technology, 2012, 252.

3. Li N, Parsons BL, Liu D, Mattoo AK.
Accumulation of wound-inducible ACC synthase transcript in tomato fruit is inhibited by salicylic acid and polyamines. Plant molecular biology. 1992 Feb 1;18(3):477-87.

4. Fan X, Matches JP, Fellowman JK. Inhibition of apple fruit aminocy clopropane-carboxylic acid oxides activity and respiration by acetylsalicylic acid [J]. Plant Physical, 1996, 149:469-471.

5. Xiaodong L, Tingting J, Feifei Z, Yu W. Advances in synthesis of acetyl salicylic acid [J]. Guangzhou chemical industry, 2019, 47(15):21-51.

6. Xiaihua C. Y2P2W(18)O(62) $\cdot \mathrm{nH} 2 \mathrm{O} / 4 \mathrm{~A}-$ Preparation of molecular sieve and catalytic synthesis of acetyl salicylic acid [J]. Chemistry notification, 2016, 79(06):528-533.

7. Gui C, Yuhui Z, Mengting T, Bailin X. Study on ultrasonic assisted synthesis of acetyl salicylic acid catalyzed by $\mathrm{SiO} 2-\mathrm{Al} 2 \mathrm{O} 3$ [J]. Journal of huaihua university, 2016, 35(5):37-40.

8. Wenquan L, Ligeng W, Ting Y, Wei S, Zheming N. Preparation and catalytic synthesis of acetylsalicylic acid by phosphotungstenmolybdenum heteropoly anion column prop hydrotalcite $[\mathrm{J}]$. Journal of zhejiang university of technology, 2011, 39(06):609-613+634.

9. Jialin W, Yingchun Z, Hong Z. The preparation of acetylsalicylic acid (aspirin) [J]. Journal of chemical intermediates, 2015, 11(1):20-21.

10. Xiaoning S, Surui Z, Qiaoling W. Synthesis of acetyl salicylic acid catalyzed by iodination of ultrasonic radiation molecules [J]. Journal of tianshui normal university, 2008(2):60-61. 